\title{
Anti-adhesive and anti-biofilm activity of NC-E08. In vitro and in vivo study using Galleria mellonella infection model
}

\author{
Gabriela F. Bombarda*, Pedro L. Rosalen, Josy G. Lazarini, Eder R. Paganini, Luis O. Regasini, Janaina C. 0. \\ Sardi
}

\begin{abstract}
Biofilms are organized microbial communities formed from an ecological succession. Biofilm formation functions as a mechanism of virulence and favors the development of diseases, including oral diseases such as dental caries and periodontal disease, in which the microorganisms Streptococcus mutans and Candida albicans are closely related. Previous studies have shown that interactions between $S$. mutans and $C$. albicans are associated with the pathogenesis of early childhood caries (ECC). Therefore, there is a great interest in finding new prototypes for antimicrobial drugs, mainly for the development of structural analogues of chalcones, which constitute one of the largest classes of natural products belonging to the flavonoid family and are considered strategic molecules for this purpose.
\end{abstract}

\section{Key words:}

Dual biofims, toxicity, nitro-chalcone.

\section{Introduction}

Biofilms are associated with many infectious diseases in humans, including those occurring in the mouth. S. mutans can efficiently cope with environmental stresses, which contributes to its ability to establish biofilms, to persist in the host, and to compete with other oral bacteria, particularly when conditions are conducive to initiation and progression of dental caries (Seneviratne et al., 2011). Interestingly, results from several clinical studies reveal that $C$. albicans is often detected in high numbers with $S$. mutans in biofilms from children with early childhood caries (He et al., 2017). In biofilms formed in vitro, the presence of $C$. albicans dramatically modifies the physical environment by increasing biomass and EPS production, thereby enhancing biofilm accumulation and stability. Biofilms mixed are considered resistant to conventional therapies and this fact has stimulated the search for alternative antimicrobial agents that can prevent biofilms formation and the onset of associated diseases. Therefore, the objective of this project was to evaluate the effect of the nitrochalcone (NC-E08) on mixed biofilms of $C$. albicans and $S$. mutans formed on hydroxyapatite disks, through cell viability (plating), Microscopy Scanning Electron (MEV), in vivo and in vitro adhesion and Galleria mellonella model infection.

Results and Discussion

Cell viability results of biofilms formed on hydroxyapatite disks treated with NC-E08, in addition to studies of their in vivo toxicity using the Galleria mellonella invertebrate model, demonstrated that NC-E08 was able to inhibit planktonic cell growth of $S$. mutans and $C$. albicans with MIC values between 15.75 and $0.97 \mu \mathrm{g} / \mathrm{mL}$, respectively. For evaluation of the cell viability and SEM, the mixed biofilms were formed on hydroxyapatite disks and were treated with NC-E08 in 3 times per day, during 120h. For the in vivo adhesion tests were used Human Gingival Fibroblast Cells (HGF-1-ATCC CRL-2014) and the evaluation of adhesion was made in 3 times (1, 2, and $3 \mathrm{~h}$ ). Moreover, for the in vivo infection was used the $G$. mellonella model, the larvae were infected with pre formed biofilm and treated with NC-E08, Chlorhexidine digluconate and artificial saliva and these were evaluated during $120 \mathrm{~h}$.
The results demonstrated that NC-E08 was able to inhibit biofilms of $C$. albicans $(25 \%)$, S. mutans $(30 \%)$, and the association of these pathogens in approximately $36 \%$ for C. albicans and $41 \%$ for S. mutans. In addition, it also showed anti-adhesive capacity in both polystyrene plates (approximately $32 \%$ for both) and in human gingival fibroblasts, where NC-E08 showed anti-adhesive capacity at all times studied. In the treatment of infection in the model G. mellonella, the NC-E08 presented protection capacity when compared to the larvae that did not receive the treatment.

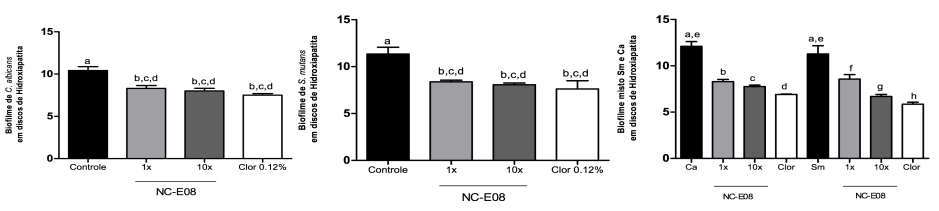

Image 1. Cell viability of biofilms of $C$. albicans, $S$. mutans and mixed of $S$. mutans and $C$. albicans treated with NC-E08(1X $\mathrm{MIC}$ ), NC-E08 (10X MIC) and digluconate of Clorexidine $0,12 \%$. $p<0.05$ - One-way ANOVA with Post Tukey test.

\section{Conclusions}

Nitrochalcone (NC-E08) demonstrated excellent antiadhesive and anti-biofilm activity on Candida albicans and Streptococcus mutans, as well as low toxicity in vivo, and a efficient activity against the in vivo G. mellonella model.

\section{Acknowledgement}

Thanks to my advisor, Janaina C. O. Sardi and to SAE UNICAMP- PIBIC

Seneviratne CJ, Zhang CF, Samaranayake LP. Dental plaque biofilm in oral health and disease. Chin J Dent Res. 2011;14(2):87-94. Review.

He J, Kim D, Zhou X, Ahn SJ, Burne RA, Richards VP, Koo H. RNA-Seq Reveals Enhanced Sugar Metabolism in Streptococcus mutans Cocultured with Candida albicans within Mixed-Species Biofilms. Front Microbiol. 2017 Jun 8;8:1036. doi: 10.3389/fmicb.2017.01036. 